\title{
Cavity light bullets in a dispersive Kerr medium
}

\author{
S. D. Jenkins, F. Prati, and L. A. Lugiato \\ CNISM and CNR-INFM, Dipartimento di Fisica e Matematica, Università dell'Insubria, Via Valleggio 11, 22100 Como, Italy \\ L. Columbo \\ Institut du Non Lineaire de Nice, UMR CNRS 6618, Universite de Nice Sophia Antipolis, 1361 Route des Lucioles, \\ F-06560 Valbonne, France \\ M. Brambilla \\ CNISM and CNR-INFM, Dipartimento di Fisica Interateneo, Università e Politecnico di Bari, Via Orabona 4, 70125 Bari, Italy
}

(Received 23 March 2009; published 22 September 2009)

\begin{abstract}
We demonstrate the existence of three-dimensional localized structures, known as cavity light bullets, that circulate in a driven cavity filled with a self-focusing dispersive medium. Though unstable, we show through a linear stability analysis how a multiphoton absorption can stabilize these structures. Bullets may be written and erased from the cavity at controlled positions, allowing them to act as elements in three-dimensional pixel arrays. We illustrate the process of writing and erasing bullets in the cavity through dynamic numerical simulations.
\end{abstract}

DOI: 10.1103/PhysRevA.80.033832

PACS number(s): 42.65.Sf, 42.65.Tg, 42.65.Hw

\section{INTRODUCTION}

Sustained self-confinement of isolated structures represents one of the most important consequences of nonlinear optical processes. The most broadly applied example, a Kerr type self-focusing nonlinearity combating dispersion in optical fibers, results in one-dimensional solitons. In higher dimensions, however, stationary solutions in the presence of such a nonlinearity tend to collapse [1].

In driven optical cavities, on the other hand, a selffocusing interaction with an intracavity medium can balance diffraction resulting in stable two-dimensional (2D) structures [2-7]. In such systems, a broad area homogeneous cw holding beam drives the cavity, sustaining a transversely uniform background field. On top of this background, localized stationary structures, or cavity solitons (CSs), can survive under the influence of various types of nonlinear interactions. While a freely propagating Kerr spatial soliton is unstable against collapse, a CS sustained in a Kerr medium can be stable against arbitrary perturbations [6]. Furthermore, one can write or erase solitons in the cavity by shining control beams with an appropriate range of amplitude, phase, and durations on the target location [8,9]. Experimental realization of CSs first occurred in extended cavities [10-12]. Later, experiments with semiconductor vertical microresonators demonstrated the use of CSs as controllable micropixels $[7,13,14]$. Extension of the cavity, allowing for more complex longitudinal dynamics, would open the door to the realization of simultaneous transverse and longitudinal localization and the formation of cavity light bullets (CLBs). These bullets have recently been predicted for cavities filled with a saturable absorber [15]. Dispersive properties of the material are not considered in that model, however, and longitudinal localization occurs only when losses due to material absorption or transmission through cavity mirrors are not negligible.

In this paper, by contrast, we demonstrate the existence of CLBs in a medium with anomalous dispersion in the low loss limit and their stability in the presence of multiphoton absorption processes. In particular, we consider a Kerr type self-focusing nonlinearity, where, analogous to transverse localization, longitudinal confinement is achieved through the interplay between nonlinear self-focusing and dispersion. In such a system, Tlidi et al. [16] demonstrated that for a range of holding beam intensities and cavity detunings, a homogeneous background can coexist with circulating threedimensional (3D) lattices, similar to hexagonal lattices which may coexist with a homogeneous background in a 2D system. Such a bistability has signaled the existence of CSs in 2D systems, and analogously we hypothesize that the moving 3D lattices indicate the existence of Kerr CLBs. We show that such bullets are supported by the system. However, like their freely propagating counterparts, these bullets are unstable only linear absorption is included in the model.

With freely propagating spatial solitons, once collapse takes hold, higher-order effects, such as multiphoton absorption, become relevant to the propagation dynamics. In fact, stationary solutions of the field exist in which a bright central peak, which suffers from nonlinear losses, is continuously fed by fat low-intensity tails $[17,18]$. In two (three) dimensions, these solutions behave asymptotically as unbalanced superpositions of (spherical) Hankel functions. Such objects have come to be known as unbalanced Bessel beams (UBBs) [17] (in the case of transverse confinement), or unbalanced $O$ waves [18] (in the 3D case with anomalous dispersion). Two dimensional UBBs have been observed in propagation in self-focusing materials such as water $[17,19]$, where multiphoton absorption operates. The spontaneous formation of ellipsoidal spectra, characteristic of $O$ waves, has also been observed [20]. Because energy must continuously flow from the tails to feed the central peak, however, the reservoir of the tails is eventually exhausted in a physical system, and these $O$ waves cannot propagate indefinitely.

In this paper, we show how multiphoton absorption processes can serve to stabilize cavity light bullets, in much the same way they stabilize freely propagating Bessel beams. 
Unlike their stable freely propagating counterparts, however, the CLBs are fully localized (with tails that exponentially approach the homogeneous background), and the losses are counterbalanced by the energy from the holding beam. Additionally, we show how these CLBs can be written and erased at specified positions in the cavity through the application of control pulse sequences. We conclude, therefore, that CLBs can serve as elements in a 3D pixel array in an optical resonator.

The remainder of this paper is organized as follows. In Sec. II we provide a theoretical description of the field dynamics. We also describe the procedure for addressing a specific point in the cavity during a write or erase process in the subsection of Sec. II. This is followed in Sec. III by a discussion of localized stationary solutions of the intracavity field (CLBs). We also perform a linear stability analysis to investigate the stability of these CLBs and examine the effects of nonlinear losses. We show that although the bullets are highly unstable in a pure Kerr medium, the inclusion of nonlinear losses stabilizes the bullets. In Sec. IV, we describe how CLBs can be written and erased at controlled positions in the cavity. We use dynamic simulations to illustrate the birth and death of CLB resulting from application of a series of write and erase pulses, respectively. Conclusions follow in Sec. V.

\section{THEORETICAL MODEL}

We consider a generalization of the model presented in Ref. [2] that accounts for anomalous intracavity dispersion and multiphoton absorption. A pump field with carrier frequency $\omega_{0}=2 \pi c / \lambda$ (where $\lambda$ is the free space wavelength) and an envelope $E_{I}\left(\vec{r}_{\perp}, t\right)$ depending on transverse position $\vec{r}_{\perp}$ and time $t$ drives a ring cavity of length $\mathcal{L}$. This field impinges on an input beam splitter with intensity transmittance $\mathcal{T} \ll 1$ at longitudinal position $z=0$. An intracavity field with a slowly varying envelope $E(\vec{r}, t)$ at position $\vec{r}=\vec{r}_{\perp}+z \hat{z}$-normalized such that $|E|^{2}$ is the root-mean-square intensity-propagates along the longitudinal $z$ axis. This field interacts with a nonlinear dispersive medium extending from the input mirror at $z=0$ to $z=L \leq \mathcal{L}$. One observes the dynamics within the cavity via the field exiting an output mirror with intensity transmittance $\mathcal{T}$ placed between the nonlinear material and the input beam splitter. Corrective optics, such as an imaging telescope, project the field image at $z=L$ onto the input face of the material at $z=0$. The envelope dynamics within the medium are governed by

$$
\begin{aligned}
\left(\frac{\partial}{\partial t}\right. & \left.+v_{g} \frac{\partial}{\partial z}\right) E(\vec{r}, t)=\left[i \frac{v_{g}}{2 k_{0}} \nabla_{\perp}^{2}\right. \\
& \left.-i \frac{v_{g} k_{0}^{\prime \prime}}{2} \frac{\partial^{2}}{\partial t^{2}}-\frac{v_{g} \alpha_{1}}{2}+f\left(|E|^{2}\right)\right] E(\vec{r}, t),
\end{aligned}
$$

where $v_{g}$ is the group velocity, $k_{0}=\omega_{0} n_{0} / c$ is the carrier wave number, $n_{0}$ is the index of refraction, $\nabla_{\perp}^{2}=\partial_{x}^{2}+\partial_{y}^{2}$ is the transverse Laplacian, $k_{0}^{\prime \prime}=d^{2} k /\left.d \omega^{2}\right|_{\omega=\omega_{0}}<0$ is the dispersion parameter, and $\alpha_{1}$ is the linear absorption coefficient. The function $f\left(|E|^{2}\right)=i\left(v_{g} \omega_{0} n_{2} / c\right)|E|^{2}-\left(v_{g} \alpha_{K} / 2\right)|E|^{2(K-1)}$ accounts for the nonlinear interaction with a material in which plasma defocusing, saturation of the nonlinearity, and delayed nonlinear effects can be neglected; $n_{2}>0$ is the nonlinear index of refraction and $\alpha_{K}(K>1)$ is the coefficient of a $K$-photon absorption process. This nonlinear absorption can result, for example, from multiphoton ionization. Such a nonlinear regime has been observed in experiments involving intense pulse propagation in water [19-21] and fused silica [22]. We handle the field dynamics of the empty space between $z=L$ and $z=\mathcal{L}$ by making the coordinate transformation $t_{a}=t$ $+(\mathcal{L} / L-1) z / c$ [23]. The transformed dynamics are governed by Eq. (1) with the substitutions $t \rightarrow t_{a}$ and $v_{g} \rightarrow V \equiv \bar{v} L / \mathcal{L}$, where $\bar{v} \equiv \mathcal{L} /\left[\left(L / v_{g}\right)+(\mathcal{L}-L) / c\right]$ is the average group velocity within the cavity. The fields then satisfy the boundary condition

$$
E\left(\vec{r}_{\perp}, z=0, t_{a}\right)=\mathcal{R} e^{-i \delta_{0}} E\left(\vec{r}_{\perp}, z=L, t_{a}\right)+\mathcal{T}^{1 / 2} E_{I}\left(\vec{r}_{\perp}, t_{a}\right),
$$

where $\delta_{0}$ is the detuning of the input beam from the nearestresonant-cavity mode, and $\mathcal{R}=1-\mathcal{T}$ is the intensity reflectance of the input and output mirrors. In these transformed coordinates, the cavity has an effective length $L$, and the cavity round trip time remains unchanged $(\mathcal{L} / \bar{v}=L / V)$. Furthermore, in the slowly varying envelope approximation, when $\left|V^{3} k_{0}^{\prime \prime} \partial^{2} E / \partial z^{2}\right|,\left|V k_{0}^{\prime \prime} \partial^{2} E / \partial t_{a}^{2}\right| \ll\left|\partial E / \partial t_{a}\right| \sim|V \partial E / \partial z|$ and linear absorption can be neglected over the length of the cavity $\left(\alpha_{1} L \ll 1\right)$, we can make the substitution $\partial^{2} E / \partial t_{a}^{2}$ $\approx V^{2} \partial^{2} E / \partial z^{2}[24]$, yielding

$$
\begin{aligned}
\left(\frac{\partial}{\partial t_{a}}\right. & \left.+V \frac{\partial}{\partial z}\right) E\left(\vec{r}, t_{a}\right)=\left[i \frac{V}{2 k_{0}} \nabla_{\perp}^{2}\right. \\
& \left.-i \frac{V^{3} k_{0}^{\prime \prime}}{2} \frac{\partial^{2}}{\partial z^{2}}-\frac{V \alpha_{1}}{2}+\frac{V}{v_{g}} f\left(|E|^{2}\right)\right] E\left(\vec{r}, t_{a}\right) .
\end{aligned}
$$

Following the treatment of Ref. [25] which considered a cavity driven by only a cw pump field, we introduce a transformed field

$$
F\left(\vec{r}, t_{a}\right)=e^{\left(\log \mathcal{R}-i \delta_{0}\right) z / L} E\left(\vec{r}, t_{a}\right)+\mathcal{T}^{1 / 2} \frac{z}{L} E_{I}\left(\vec{r}_{\perp}, t_{a}+\delta t_{a}(z)\right),
$$

where $\delta t_{a}(z) \equiv(L-z) / V$ is the delay until a point at $z$ propagating through the medium completes a round trip through the cavity. With this transformation, cavity losses and driving field effects, which were previously accounted for by the boundary condition in $E$ [Eq. (2)], manifest themselves in the dynamic equation for the field $F$, and the boundary condition becomes periodic,

$$
F\left(\vec{r}_{\perp}, 0, t_{a}\right)=F\left(\vec{r}_{\perp}, L, t_{a}\right)
$$

The dynamics of this transformed field are given by 


$$
\begin{aligned}
\left(\frac{\partial}{\partial t_{a}}+V \frac{\partial}{\partial z}\right) F\left(\vec{r}, t_{a}\right) \\
=\frac{\Gamma}{2} e^{\left(\log \mathcal{R}-i \delta_{0}\right) z / L}\left[i r_{0}^{2} \nabla_{\perp}^{2}+i z_{0}^{2} \frac{\partial^{2}}{\partial z^{2}}-(1+i \theta)\right. \\
\left.+i \frac{|E|^{2}}{F_{0}^{2}}-\frac{\beta_{K}}{2}\left(\frac{|E|^{2}}{F_{0}^{2}}\right)^{K-1}\right] E\left(\vec{r}, t_{a}\right) \\
+\frac{\kappa}{2} \frac{\mathcal{T}}{|\log \mathcal{R}|} \mathcal{T}^{-1 / 2} E_{I}\left(\vec{r}_{\perp}, t_{a}+\delta t_{a}(z)\right),
\end{aligned}
$$

where $\Gamma \equiv \kappa+V \alpha_{1}$ is the linear intensity decay rate, $\kappa$ $\equiv 2 V|\log \mathcal{R}| / L$ is the cavity decay rate, $V \alpha_{1}$ is the portion of decay due to linear absorption, $r_{0} \equiv\left(V / k_{0} \Gamma\right)^{1 / 2}$ and $z_{0}$ $\equiv\left[V^{3} k_{0}^{\prime \prime} / \Gamma\right]^{1 / 2}$ are transverse and longitudinal length scales, respectively, $F_{0}^{2} \equiv c \Gamma /\left(2 V \omega_{0} n_{2}\right)$ is the characteristic intensity, $\theta \equiv(\kappa / \Gamma)\left(\delta_{0} / \mathcal{T}\right)$ is the detuning parameter, and $\beta_{K}$ $\equiv 2 V \alpha_{K}\left|F_{0}\right|^{2(K-1)} / \Gamma$ is the normalized nonlinear loss rate.

The transformation of Eq. (4) differs from that of Ref. [25] in that the time argument of $E_{I}$ in the latter does not account for the time needed to complete a round trip through the cavity. When the pump field varies slowly with respect to the cavity round trip time, our transformation is equivalent to that considered in Ref. [25]. However, as we will discuss in the subsection of Sec. II, the task of writing and erasing CLBs at controlled positions requires the injection of pulses shorter than the cavity, and the inclusion of $\delta t_{a}(z)$ in Eq. (4) simplifies the treatment of the intracavity dynamics.

In this paper, we consider the field dynamics in the limits of small detuning, where $\left|\delta_{0}\right| \ll 1$, and low loss, where the loss rate $\Gamma$ is much smaller than the free spectral range $V / L$. Recalling that $\Gamma /(V / L)=2|\log \mathcal{R}|+\alpha_{1} L$, we see that in addition to the small linear loss condition $\left(\alpha_{1} L \ll 1\right)$ assumed in the derivation leading to Eq. (3), the limit of low loss requires a small mirror transmittance, $\mathcal{T} \ll 1$. This regime is consistent with the so-called mean-field limit [23]. These limits allow us to make several simplifying approximations on Eq. (6).

Assuming that $\mathcal{T}^{-1 / 2}\left|E_{I}\right| \sim|F|$ and that the intracavity field and the pump field vary over similar length and time scales, we have $e^{\left(\log \mathcal{R}-i \delta_{0}\right) z / L} E \approx F, \quad e^{\left(\log \mathcal{R}-i \delta_{0}\right) z / L} \nabla_{\perp}^{2} E \approx \nabla_{\perp}^{2} F$, and $e^{\left(\log \mathcal{R}-i \delta_{0}\right) z / L}\left(\partial^{2} E / \partial z^{2}\right) \approx \partial^{2} F / \partial z^{2}$. The equation of motion for the intracavity field then simplifies to

$$
\begin{aligned}
\left(\frac{\partial}{\partial t_{a}}+\right. & \left.+\frac{\partial}{\partial z}\right) F\left(\vec{r}, t_{a}\right) \\
& =\frac{\Gamma}{2}\left[i r_{0}^{2} \nabla_{\perp}^{2}+i z_{0}^{2} \frac{\partial^{2}}{\partial z^{2}}-(1+i \theta)\right. \\
& \left.+i \frac{|F|^{2}}{F_{0}^{2}}-\frac{\beta_{K}}{2}\left(\frac{|F|^{2}}{F_{0}^{2}}\right)^{K-1}\right] F\left(\vec{r}, t_{a}\right) \\
& +\frac{\kappa}{2} \mathcal{T}^{-1 / 2} E_{I}\left(\vec{r}_{\perp}, t_{a}+\delta t_{a}(z)\right),
\end{aligned}
$$

Recognizing that Eq. (7) represents the dynamics of structures which travel through the cavity at group velocity $V$, and observing that the temporal argument of the driving term $t_{a}$ $+\delta t_{a}(z)=t_{a}+(L-z) / V=(L / V)-\left(z-V t_{a}\right) / V$, it seems natural to examine these dynamics in a moving frame of coordinates. We must take care, however, to define these moving coordinates in such a way that they are consistent with the finite length of the cavity $(0 \leq z<L)$. With this in mind, we introduce the moving frame position $\vec{r}_{V}=\vec{r}_{\perp}+z_{V} \hat{z}$ with $z_{V}$ $\equiv z-V t_{a}+\tilde{n}\left(z, t_{a}\right) L$, where $\tilde{n}\left(z, t_{a}\right) \equiv\left\lfloor\left(V t_{a}-z\right) / L\right\rfloor+1$ is the number of times a structure initially at position $z$ at time zero strikes the input mirror. Here, $\lfloor x\rfloor$ is the largest integer less than or equal to $x$. Adding this integer number of cavity lengths to the "traditional" moving frame coordinate $z-V t_{a}$ ensures that $z_{V}$ lies in the interval $[0, L)$ and that the fixed interval $[0, L)$ in the moving frame can unambiguously be mapped back to physical positions $0 \leq z<L$ in the laboratory fixed frame. Because the field satisfies the periodic boundary condition of Eq. (5), its dynamics can be described in the moving frame of coordinates by

$$
\begin{aligned}
\frac{\partial F}{\partial t_{a}}= & \frac{\Gamma}{2}\left[i r_{0}^{2} \nabla_{\perp}^{2}+i z_{0}^{2} \frac{\partial^{2}}{\partial z_{V}^{2}}-(1+i \theta)+i \frac{|F|^{2}}{F_{0}^{2}}\right. \\
& \left.-\frac{\beta_{K}}{2}\left(\frac{|F|^{2}}{F_{0}^{2}}\right)^{K-1}\right] F\left(\vec{r}, t_{a}\right)+\frac{\kappa}{2} \mathcal{T}^{-1 / 2} E_{I}\left(\vec{r}_{\perp}, \varsigma\right) .
\end{aligned}
$$

The time argument $t_{a}+\delta t_{a}(z)$ of $E_{I}$ has been expressed in terms of the moving frame coordinates as

$$
\varsigma \equiv \frac{L}{V} \widetilde{n}\left(z_{V}, t_{a}\right)+\frac{L-z_{V}}{V},
$$

taking into account the inverse relation $z=z_{V}+V t_{a}$ $-\widetilde{n}\left(z_{V}, t_{a}\right) L$. In the following subsection, we will describe how variations of the driving field can be used to write or erase CLBs at controlled positions in the moving frame of reference.

\section{Pump field and addressing intracavity positions}

A uniform cw holding beam $Y_{0}$ feeds the system and sustains an intracavity homogeneous background and any CLBs that may be circulating. One can write or erase CLBs at controlled positions within the cavity by superposing sequences of control pulses onto the holding beam. With a judicious choice of control field parameters, these pulses will either reinforce, or destructively interfere with a circulating structure, resulting in the creation or erasure of a CLB. In this section, we describe the nature of these control sequences in detail. We show that they can be used to address specific points in the moving reference frame, allowing for the creation and destruction of CLBs at predetermined positions.

To better understand how to address a CLB at a specific position, let us first examine how one writes or erases a two-dimensional cavity soliton when the cavity's longitudinal degree of freedom can be ignored. Suppose one wants to write a CS at position $\vec{r}_{\perp}^{\text {(CS) }}$. To accomplish this, one would send in a control pulse of the form $E^{(\mathrm{CS})}\left(\vec{r}_{\perp}, t_{a}\right)$ $=G^{(\mathrm{CS})}\left(t_{a}\right) \varphi^{(\mathrm{CS})}\left(\vec{r}_{\perp}-\vec{r}_{\perp}^{(\mathrm{CS})}\right)$, where $\varphi^{(\mathrm{CS})}$ is a two-dimensional localized function with transverse dimensions similar to those of a CS (e.g., a 2D Gaussian with a standard deviation 
of the intensity profile $\left.\sigma_{\mathrm{CS}}\right)$. The function $\varphi^{(\mathrm{CS})}$, whose argument addresses the position $\vec{r}_{\perp}^{(\mathrm{CS})}$, is modulated in time by the envelope $G^{(\mathrm{CS})}\left(t_{a}\right)$. Whether the CS is written or erased at that position depends on the phase of the input field. For an appropriate choice of phase, width $\sigma_{\mathrm{CS}}$, and duration, the pulse creates a CS at position $\vec{r}_{\perp}^{(\mathrm{CS})}$. Similarly, a CS can be erased by sending in a pulse of the opposite phase.

To write or erase cavity light bullets, we must address their longitudinal positions as well as their transverse locations. We express the pump field as $E_{I}\left(\vec{r}_{\perp}, t_{a}\right)=E_{0}$ $+\sum_{\nu} E^{(\nu)}\left(\vec{r}_{\perp}, t_{a}\right)$, where the fields $E^{(\nu)}\left(\vec{r}_{\perp}, t_{a}\right)$ represent control pulse sequences labeled by an index $\nu$. Each control sequence $E^{(\nu)}\left(\vec{r}_{\perp}, t_{a}\right)$ serves to write or erase a single bullet at a specific position $\vec{r}_{V}^{(\nu)}=\vec{r}_{\perp}^{(\nu)}+z_{V}^{(\nu)} \hat{z}$ in the moving frame of coordinates. In the laboratory fixed frame (with time measured with respect to the temporal coordinate $t_{a}$ ), a structure at this point propagates through the cavity at velocity $V$, and encounters the input mirror at times

$$
t_{m}^{(\nu)}=\frac{L}{V} m+\frac{L-z_{V}^{(\nu)}}{V}, \quad m=0, \pm 1, \pm 2, \ldots
$$

One could imagine writing a CLB at a position $\vec{r}_{V}^{(\nu)}$ by sending a series of pulses centered at times $t_{m}^{(\nu)}$ focused on the input mirror at transverse position $\vec{r}_{\perp}^{(\nu)}$. These pulses would have roughly the same width and duration as a CLB. If the pulses in this sequence have an appropriate phase, then every time a nascent CLB completes a cavity round trip, it would be reinforced by a pulse in the control sequence as it strikes the input mirror. Likewise, a CLB can be erased by sending a similar series of pulses with the opposite phase, thereby destroying the bullet. By sending in multiple control fields (each of which is composed of a sequence of pulses) with different arrival times focused at different transverse positions, one could independently address multiple positions in the moving reference frame.

We mathematically represent a control sequence $\nu$ which addresses the moving frame position $\vec{r}_{V}^{(\nu)}$ in a manner similar to that used to express control pulses for CSs in planar cavities,

$$
E^{(\nu)}\left(\vec{r}_{\perp}, t_{a}\right)=G^{(\nu)}\left(t_{a}\right) \Xi^{(\nu)}\left(\vec{r}_{\perp}, t_{a}\right),
$$

where

$$
\Xi^{(\nu)}\left(\vec{r}_{\perp}, t_{a}\right) \equiv \sum_{m=-\infty}^{\infty} \varphi^{(\nu)}\left(\vec{r}_{\perp}-\vec{r}_{\perp}^{(\nu)}, t_{a}-t_{m}^{(\nu)}\right)
$$

represents a sequence of pulses addressing the moving frame position $\vec{r}_{V}^{(\nu)}$, and $G^{(\nu)}\left(t_{a}\right)$ is a temporal modulating function which we assume varies slowly with respect to a cavity round trip. The pulse profiles $\varphi^{(\nu)}$ have width and duration comparable to those of a CLB. Figure 1(a) illustrates the time dependence of a sample pulse sequence, where an address pulse train is modulated by a Gaussian function $G^{(\nu)}$.

By examining Eq. (8), we see that the control sequence $E^{(\nu)}$ adds a driving term to the dynamic equation of motion proportional to
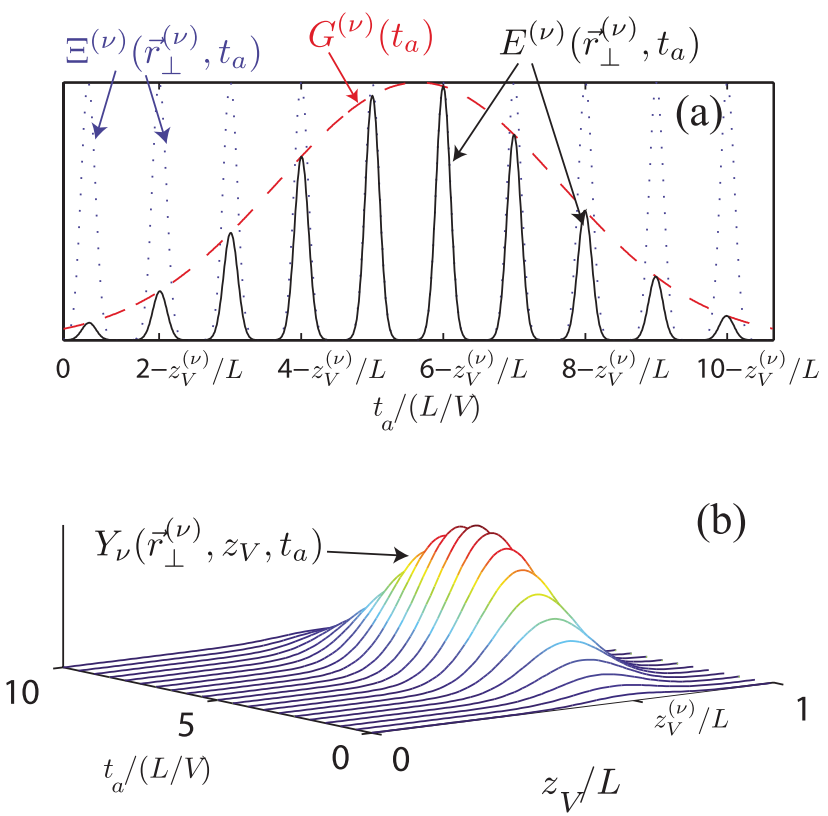

FIG. 1. (Color online) Illustrates a sample control pulse sequence $\nu$ which addresses a point $\vec{r}_{V}^{(\nu)}=\vec{r}_{\perp}^{(\nu)}+z_{V}^{(\nu)} \hat{z}$ in the moving reference frame. Panel (a) shows the profile $E^{(\nu)}\left(\vec{r}_{\perp}, t_{a}\right)$ (solid line) evaluated at $\vec{r}_{\perp}=\vec{r}_{\perp}^{(\nu)}$ of a train of pulses arriving at the input mirror at times $t_{m}^{(\nu)}=(L / V)\left(1+m-z_{V}^{(\nu)} / L\right)$ [see Eq. (10)]. This sequence is composed of a periodic pulse train $\Xi^{(\nu)}\left(\vec{r}_{\perp}, t_{a}\right)$ (dotted line) modulated by $G^{(\nu)}\left(t_{a}\right)$ (dashed line). Panel (b) shows the corresponding driving term $Y_{\nu}\left(\vec{r}_{\perp}, z_{V}, t\right)$ (evaluated at $\left.\vec{r}_{\perp}=\vec{r}_{\perp}^{(\nu)}\right)$ appearing in the equation of motion for the intracavity field in the moving reference frame [Eq. (16)].

$$
E^{(\nu)}\left(\vec{r}_{\perp}, \varsigma\right)=G^{(\nu)}(\varsigma) \sum_{m=-\infty}^{\infty} \varphi^{(\nu)}\left(\vec{r}_{\perp}-\vec{r}_{\perp}^{(\nu)}, \varsigma-t_{m}^{(\nu)}\right)
$$

where $s$ depends on both $t_{a}$ and the moving frame longitudinal position $z_{V}$ [see Eq. (9)]. Because $G^{(\nu)}$ varies slowly over a cavity round trip, however, we observe that $G^{(\nu)}(\varsigma)$ $\approx G^{(\nu)}\left(t_{a}\right)$. Moreover, taking Eq. (10) into account, we see that the temporal argument of the functions $\varphi^{(\nu)}$ can be written as

$$
\varsigma-t_{m}^{(\nu)}=(\tilde{n}-m) L / V-\left(z_{V}-z_{V}^{(\nu)}\right) / V .
$$

For our purposes, we assume the temporal extent of the individual pulse profiles $\varphi^{(\nu)}$ is much less than a cavity round trip time. This implies that $\varphi^{(\nu)}\left(\vec{r}_{\perp}-\vec{r}_{\perp}^{(\nu)}, \varsigma-t_{m}^{(\nu)}\right)$ $\approx \delta_{m, \tilde{n}} \varphi^{(\nu)}\left(\vec{r}_{\perp}-\vec{r}_{\perp}^{(\nu)},-\left(z_{V}-z_{V}^{(\nu)}\right) / V\right)$, and as a consequence

$$
E^{(\nu)}\left(\vec{r}_{\perp}, \mathrm{\varsigma}\right) \approx G^{(\nu)}\left(t_{a}\right) \varphi^{(\nu)}\left(\vec{r}_{\perp}-\vec{r}_{\perp}^{(\nu)},-\left(z_{V}-z_{V}^{(\nu)}\right) / V\right) .
$$

We see, therefore, that the sequence of pulses $\Xi^{(\nu)}\left(\vec{r}_{\perp}, t_{a}\right)$ addresses the three-dimensional position $\vec{r}_{V}^{(\nu)}$ in much the same way that the function $\varphi^{(\mathrm{CS})}\left(\vec{r}_{\perp}-\vec{r}_{\perp}^{(\mathrm{CS})}\right)$ addresses the transverse cavity soliton position $\vec{r}_{\perp}^{(\mathrm{CS})}$ in systems lacking a longitudinal degree of freedom. With this in mind, we may write the equation of motion under the influence of the pump field as 


$$
\begin{aligned}
\frac{\partial}{\partial t_{a}} F\left(\vec{r}_{V}, t_{a}\right)= & \frac{\Gamma}{2}\left[i r_{0}^{2} \nabla_{\perp}^{2}+i z_{0}^{2} \frac{\partial^{2}}{\partial z_{V}^{2}}-(1+i \theta)\right. \\
& \left.+i \frac{|F|^{2}}{F_{0}^{2}}-\frac{\beta_{K}}{2}\left(\frac{|F|^{2}}{F_{0}^{2}}\right)^{K-1}\right] F\left(\vec{r}_{V}, t_{a}\right) \\
& +\frac{\kappa}{2}\left[Y_{0}+\sum_{\nu} Y_{\nu}\left(\vec{r}_{\perp}, z_{V}, t_{a}\right)\right] .
\end{aligned}
$$

where $Y_{0} \equiv E_{0} \mathcal{T}^{-1 / 2}$, and $Y_{\nu}\left(\vec{r}_{\perp}, z_{V}, t_{a}\right) \equiv \mathcal{T}^{-1 / 2} G^{(\nu)}\left(t_{a}\right) \varphi^{(\nu)}\left(\vec{r}_{\perp}\right.$ $\left.-\vec{r}_{\perp}^{(\nu)},-\left(z_{V}-z_{V}^{(\nu)}\right) / V\right)$. Figure 1(b) illustrates the longitudinal and temporal behavior of the driving term $Y_{\nu}\left(\vec{r}_{\perp}, z_{V}, t_{a}\right)$ that corresponds to the control sequence $E^{(\nu)}\left(\vec{r}_{\perp}^{(\nu)}, t_{a}\right)$ illustrated in Fig. 1(a).

We further simplify the dynamic equation by scaling the time to the total linear decay rate, defining $\tau \equiv \Gamma t_{a} / 2$, and introducing the scaled coordinates: $\vec{\rho}_{\perp} \equiv \vec{r}_{\perp} / r_{0}$, and $\zeta$ $\equiv z_{V} / z_{0}$. Likewise, we define the scaled fields $\Phi=F / F_{0}, \Upsilon_{0}$ $=(\kappa / \Gamma) Y_{0} / F_{0}$, and $Y_{\nu}=(\kappa / \Gamma) Y_{\nu} / F_{0}$. We then find the dimensionless equation of motion

$$
\begin{aligned}
\frac{\partial \Phi}{\partial \tau}= & {\left[-(1+i \theta)+i \nabla^{2}+i|\Phi|^{2}\right] \Phi } \\
& -\frac{\beta_{K}}{2}|\Phi|^{2(K-1)} \Phi+\Upsilon_{0}+\sum_{\nu} \Upsilon_{\nu}(\vec{\rho}, \tau),
\end{aligned}
$$

where the dimensionless three-dimensional position in the moving frame $\vec{\rho}=\vec{\rho}_{\perp}+\zeta \hat{z}$, and $\nabla^{2}$ is $3 \mathrm{D}$ dimensionless Laplacian. With a constant pump field and in the absence of nonlinear losses, Eq. (17) reduces to the model considered by Tlidi et al. [16], which is, in turn, a generalization of the 2D model of Ref. [2]. Furthermore, when $\beta_{K}=0$ and one transverse dimension is frozen out (e.g., by a planar waveguide), Eq. (17) is mathematically equivalent to the dynamic equation which supports stable cavity solitons in $2 \mathrm{D}$ [6]. We therefore conclude that 2D CLBs exist and can be written and erased in the manner we described above. In Sec. III, we answer the question as to whether localized structures exist in the extension to 3D systems. The answer is yes, they exist, and they can become stable against symmetric perturbations when nonlinear losses are included. In Sec. IV, we apply the ideas of this section to illustrate through dynamical simulations how appropriate control pulse sequences can be used to write and erase CLBs at specific positions within the cavity.

\section{STATIONARY SOLUTIONS: CAVITY LIGHT BULLETS AND THEIR STABILITY}

In this section we consider stationary configurations of the field when the cavity is pumped by only the holding beam. This holding beam sustains a constant homogeneous intracavity background field on top of which cavity light bullets may circulate. Through linear stability analysis, we find these stationary CLB solutions and consider their stability against arbitrary small perturbations. We show that nonlinear absorption can serve to stabilize some of these solutions against symmetric perturbations which could otherwise instigate collapse.
The simplest of the stationary configurations is the trivial homogeneous background solution of Eq. (17) $\Phi_{0}$, which satisfies

$$
\Phi_{0}=\frac{\Upsilon_{0}}{1+\frac{\beta_{K}}{2} I_{0}^{K-1}+i\left(\theta-I_{0}\right)},
$$

where $I_{0} \equiv\left|\Phi_{0}\right|^{2}$ solves the polynomial equation

$$
I_{0}^{3}-2 \theta I_{0}^{2}+\left(\theta^{2}+1\right) I_{0}+\beta_{K} I_{0}^{K}+\frac{\beta_{K}^{2}}{4} I_{0}^{2 K-1}=\left|\Upsilon_{0}\right|^{2} .
$$

Following the treatment of Ref. [2], one can determine the stability of this homogeneous background by expanding a perturbation $\delta \Phi$ into its Fourier components $\delta \Phi_{\vec{k}}$ indexed by the wave vector $\vec{k}$. Linearizing Eq. (17) around $\Phi_{0}$, we see that each Fourier component evolves independently according to $\partial / \partial \tau\left(\delta \Phi_{\vec{k}}, \delta \Phi_{\vec{k}}^{*}\right)^{T}=\mathbf{A}_{k}\left(\delta \Phi_{\vec{k}}, \delta \Phi_{\vec{k}}^{*}\right)^{T}$ for some matrix $\mathbf{A}_{k}$, where ${ }^{T}$ indicates the transpose. The growth rate of that spectral component is given by the maximum real part of the eigenvalues of $\mathbf{A}_{k}$. For a given set of system parameters $\left\{I_{0}, \theta, K, \beta_{K}\right\}$, when the maximum real part $\Lambda_{b g}$ of these eigenvalues taken over all possible wave numbers $k$ is negative, the homogeneous background is stable. If we limit ourselves to the regime $I_{0}>\theta / 2$, as we do throughout this paper, $\Lambda_{b g}$ is given by

$$
\Lambda_{b g}=\sqrt{I_{0}^{2}+(K-1)^{2} \frac{\beta_{K}^{2}}{4} I_{0}^{2(K-1)}}-1-K \frac{\beta_{K}}{2} I_{0}^{K-1} .
$$

The background is dynamically stable when $I_{0} \leq 1+O\left(\beta_{K}\right)$ [2].

CLBs exist as localized modulations on top of the homogeneous background. For simplicity, we limit our discussion to a regime where $I_{0}$ is a single valued function of $\left|Y_{0}\right|^{2}:|\theta|<\sqrt{3}$. We define the displacement from the homogeneous field $\phi(\vec{\rho}, \tau)$ such that $\Phi(\vec{\rho}, \tau)=\Phi_{0}(1+\phi)$, which satisfies the equation

$$
\frac{\partial \phi}{\partial \tau}=Q\left(\phi, \phi^{*}\right)
$$

where

$$
\begin{aligned}
Q\left(\phi, \phi^{*}\right)= & {\left[-(1+i \theta)+i \nabla^{2}\right] \phi(\vec{\rho}, t) } \\
& +\left[i I(\vec{\rho}, \tau)-\frac{\beta_{K}}{2} I(\vec{\rho}, \tau)^{K-1}\right][1+\phi(\vec{\rho}, t)] \\
& -i I_{0}+\frac{\beta_{K}}{2} I_{0}^{K-1}
\end{aligned}
$$

and the intensity at position $\vec{\rho}$ is $I(\vec{\rho}, t) \equiv I_{0}|1+\phi(\vec{\rho}, t)|^{2}$. The field $\phi$ satisfies the periodic boundary conditions $\phi\left(\vec{\rho}_{\perp}, L / z_{0}, \tau\right)=\phi\left(\vec{\rho}_{\perp}, 0, \tau\right)$. When $L \gg z_{0}$, we can neglect boundary effects, and Eq. (21) supports localized solutions that possess spherical symmetry. We therefore seek stationary CLBs centered at some dimensionless position $\vec{\rho}^{(c)}$ in the moving frame which depend only on a single variable $\rho$ $\equiv\left|\vec{\rho}-\vec{\rho}^{(c)}\right|$.

For a given set of parameters $\left\{I_{0}, \theta, K, \beta_{K}\right\}$, one can find stationary CLBs $\phi_{S}(\rho)$ by setting the time derivative in Eq. 
(21) to zero and solving the resulting ordinary differential equation in $\rho$. For simplicity, we define the symmetric function $u_{S} \equiv \rho \phi_{S}$ so that the Laplacian undergoes the simplification $\rho \nabla^{2} \phi_{S}=d^{2} u_{S} / d \rho^{2}$. This function satisfies

$$
Q\left(\frac{u_{S}}{\rho}, \frac{u_{S}^{*}}{\rho}\right)=0 .
$$

This static equation is subject to the boundary conditions $d u_{S} /\left.d \rho\right|_{\rho=0}=\lim _{\rho \rightarrow 0} u_{S} / \rho$, and $\lim _{\rho \rightarrow \infty} u_{S}(\rho)=0$. The former condition is implied by the assumption that $\phi_{S}(\rho)$ is differentiable at $\rho=0$. We solve this system using the shooting procedure described in Refs. $[3,4]$. In this procedure, we exploit the boundary condition for $\rho \gg 1$ by assuming $\mid u_{S}(\rho$ $\gg 1) \mid \ll 1$ and expanding Eq. (23) to first order in $u_{S}$ and $u_{S}^{*}$, neglecting higher-order terms. In this region, one can check that the solution of Eq. (23) has the form

$$
u_{S}(\rho \gg 1)=e^{-\gamma \rho}\left(A X_{-} e^{-i \tilde{k} \rho}+A^{*} X_{+} e^{\tilde{i k \rho}}\right),
$$

where $A$ is an arbitrary complex constant. The decay coefficient of the tails $\gamma$, the wave number of their oscillation $\tilde{k}$, and the relative weight factors $X_{+}$and $X_{-}$are fixed by the system parameters $I_{0}, \theta, K$, and $\beta_{K}$. Equation (24) indicates that any symmetric stationary bullet must be localized about its center with tails that decay as $\left|u_{S}\right| \sim \exp (-\gamma \rho)$. To find a solution to the full nonlinear equation [Eq. (23)] we choose a $\rho_{\max } \gg 1$, and take $u_{S}\left(\rho_{\text {max }}\right)$ given in Eq. (24) as the solution at $\rho_{\max }$. By varying the complex free parameter $A$ and numerically integrating Eq. (23), we search for a solution that satisfies the boundary condition $\lim _{\rho \rightarrow 0} d u_{S} / d \rho$ $=\lim _{\rho \rightarrow 0} u_{S} / \rho$. In practice, we shoot for the boundary condition $u_{S}\left(\rho_{\min }\right) / \rho_{\min }=d u_{S} /\left.d \rho\right|_{\rho=\rho_{\min }}$ for some $\rho_{\min } \ll 1$.

By examining localized stationary solutions of Eq. (21), one observes that nonlinear losses strongly influence the CLB spatial and spectral profiles. Panels (a) and (c) of Fig. 2 illustrate the intensity profiles of a stationary spherically symmetric solution both in the absence and in the presence of nonlinear losses. We also compare the spectra $S(\vec{k})$ $\equiv(2 \pi)^{-3}\left|\int d^{3} \rho \phi(\vec{\rho}) e^{-i \vec{k} \cdot\left(\vec{\rho}-\vec{\rho}^{(c)}\right)}\right|^{2}$ for these two solutions in panels (b) and (d). In the absence of nonlinear absorption, the CLB has a broad spherically symmetric spectrum peaked at the carrier wave number (i.e., where $|\vec{k}|=0$ ). On the other hand, when losses are included the solution has a much less pronounced central component and the bulk of the energy occupies a spherical shell. Thus, the multiphoton absorption gives the CLB spectral properties very similar to freely propagating nonlinear $O$ waves [18].

Our ultimate goal is to determine the dynamic stability of these spherically symmetric solutions. To that end, we analyze the stability of CLBs against arbitrary disturbances in three dimensions by considering the evolution of the perturbed field $\phi_{S}(\rho)+\delta \phi(\vec{\rho}, \tau)$. We expand the perturbation in terms of spherical harmonics $Y_{\ell, m}$ as $\delta \phi$ $=\sum_{\ell, m} \psi_{\ell, m}(\rho, \tau) Y_{\ell m}(\hat{\rho})$, where $\hat{\rho} \equiv\left(\vec{\rho}-\vec{\rho}^{(c)}\right) / \rho$ is the unit vector indicating the orientation of $\vec{\rho}-\vec{\rho}^{(c)}$, and the functions $\psi_{\ell, m}(\rho)(\ell=0,1,2, \ldots ; m=-\ell,-\ell+1, \ldots, \ell)$ give the radial dependence of $2^{\ell}$-pole perturbations $\left(2^{0}\right.$-pole $\equiv$ monopole, $2^{1}$-pole $\equiv$ dipole, $2^{2}$-pole $\equiv$ quadrupole, etc.). We estimate the
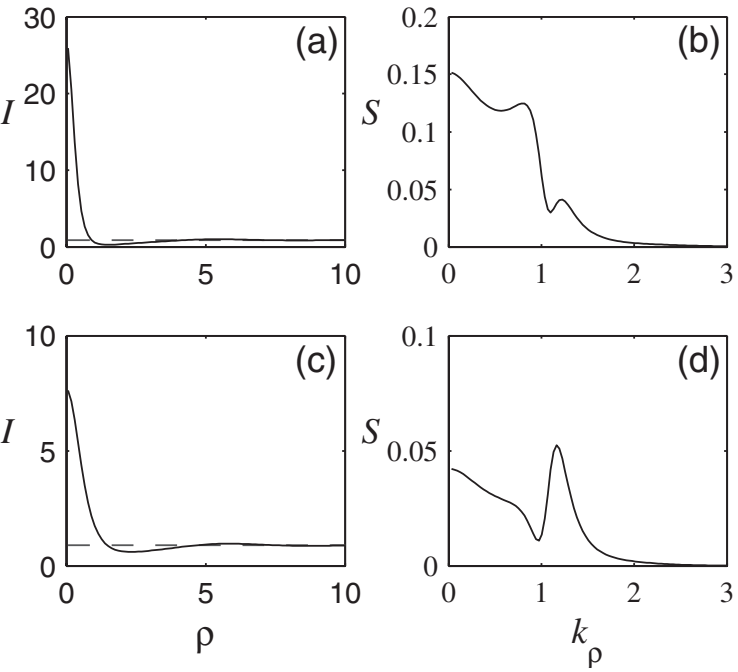

FIG. 2. Spatial (a), (c) and spectral (b), (d) profiles of spherically symmetric CLBs in the absence of nonlinear losses (a), (b), $\beta_{K}=0$, and in the presence of (c), (d) nonlinear losses $\beta_{K}=10^{-4}$. The dashed line in plots (a) and (c) represents the background intensity. In all plots, the background intensity $I_{0}=0.9$ and $\theta=0.7$.

dynamics of the functions $\psi_{\ell, m}(\rho)$ by expanding the dynamic equation [Eq. (21)] to first order in the terms $\psi_{\ell, m}(\rho) Y_{\ell, m}(\hat{\rho})$ and $\psi_{\ell, m}^{*}(\rho) Y_{\ell, m}^{*}(\hat{\rho})$. Since the spherical harmonics are orthonormal and their complex conjugates have the property $Y_{\ell, m}^{*}(\hat{\rho})=(-1)^{m} Y_{\ell,-m}(\hat{\rho})$, we find that the various multipole perturbations evolve according to

$$
\frac{\partial}{\partial \tau}\left(\begin{array}{c}
\psi_{\ell, m} \\
(-1)^{m} \psi_{\ell,-m}^{*}
\end{array}\right)=\mathcal{J}_{\ell}\left(\begin{array}{c}
\psi_{\ell, m} \\
(-1)^{m} \psi_{\ell,-m}^{*}
\end{array}\right),
$$

where the linear operator $\mathcal{J}_{\ell}$ depends on $\ell$ through the action of the Laplacian on the $2^{\ell}$-pole contributions to the perturbation, $\mathcal{D}_{\ell}=\partial^{2} / \partial \rho^{2}+(2 / \rho) \partial / \partial \rho-\ell(\ell+1) / \rho^{2}$. Since the linearized system dynamics are rotationally invariant, the stability properties of the CLB are independent of the orientation of the multipole perturbations, and hence $\mathcal{J}_{\ell}$ is independent of $m$. We characterize the stability of a bullet through the parameters $\Lambda_{\ell}(\ell=0,1,2, \ldots)$, which we define to be the maximum real part of the eigenvalues of $\mathcal{J}_{\ell}$. A CLB is stable against $2^{\ell}$-pole disturbances if $\Lambda_{\ell}<0$, and is unstable against these perturbations if $\Lambda_{\ell}>0$. In numerically determining $\Lambda_{\ell}$, we approximated the operator $\mathcal{J}_{\ell}$ using the discrete Hankel transform technique discussed in Refs. $[26,27]$.

We first examine CLB solutions in the absence of nonlinear losses. Figure 3(a) shows the maximum intensity of a CLB as a function of the background intensity for a fixed detuning $\theta=0.7$. We see, in a manner analogous to Kerr CSs [6], that for a given background intensity, there are two soliton branches with different peak intensities. These threedimensional Kerr CLBs, however, tend to be much more intense than their two-dimensional counterparts. We show the results of our linear stability analysis in Figs. 3(b) and 4. Panel (b) of Fig. 3 shows the stability parameter, $\Lambda_{0}$ for spherically symmetric (monopole) perturbations. The posi- 

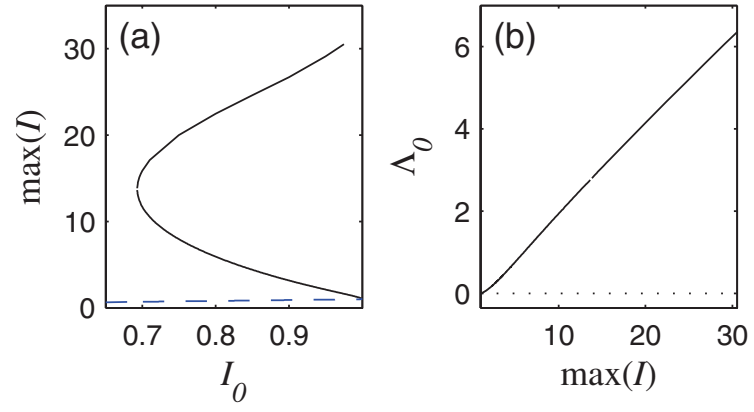

FIG. 3. (Color online) The peak intensity (a) and stability parameter against spherically symmetric perturbations $\Lambda_{0}$ (b) of a Kerr cavity light bullet in the absence of nonlinear losses $\left(\beta_{K}=0\right)$. Panel (a) illustrates that for a given background intensity there are both high intensity (upper branch) and low-intensity (lower branch) solutions. The parameter $\Lambda_{0}>0$ plotted against peak CLB intensity in panel (b) illustrates the instability of the Kerr CLBs against spherically symmetric perturbations. The dashed line in panel (a) represents the intensity of the homogeneous background. The detuning parameter $\theta=0.7$.

tive values of $\Lambda_{0}$ indicate that these CLBs are always unstable, and further, the degree to which they are unstable increases with the peak intensity. This is in sharp contrast to the two-dimensional case where, although the lower branch is unstable against cylindrically symmetric perturbations, the upper branch can be stable for appropriate values of $I_{0}$ and $\theta$ [6].

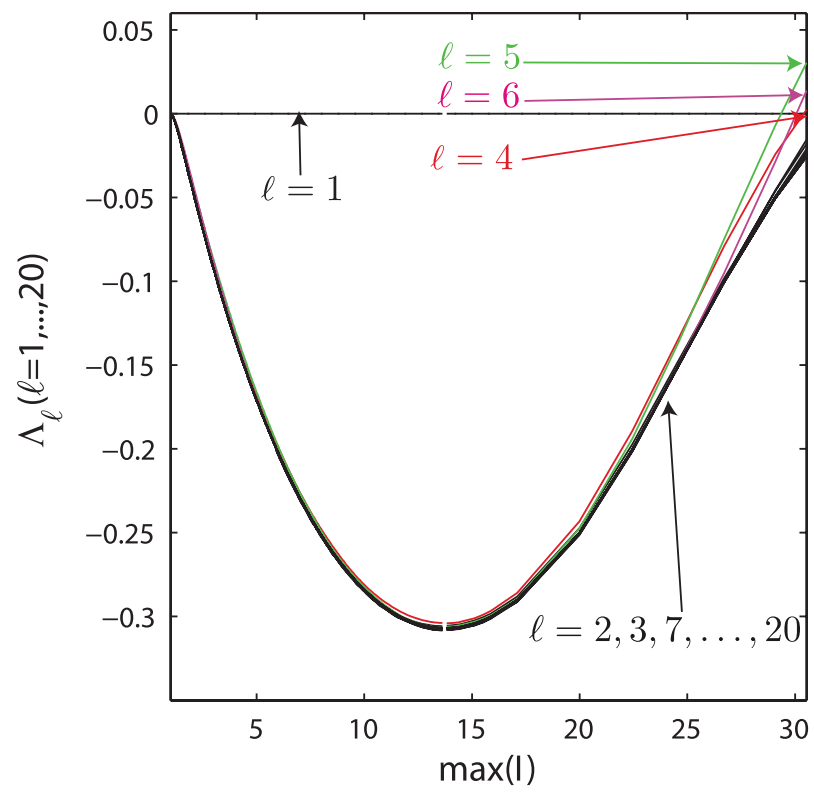

FIG. 4. (Color online) The stability parameters $\Lambda_{\ell}$ of a Kerr cavity light bullet against multipole disturbances $(\ell=1,2, \ldots, 20)$ as a function of peak CLB intensity in the absence of nonlinear losses $\left(\beta_{K}=0\right)$. The displayed value of $\Lambda_{1}$ shows that the CLBs are marginally stable $\left(\Lambda_{1}=0\right)$ against dipolar disturbances, corresponding to translations. Stability parameters $\Lambda_{\ell}$ for $\ell=2,3,7, \ldots, 20$ closely follow that of the background $\Lambda_{b g}$ on which the CLBs reside, and the bullets are stable against the corresponding $2^{\ell}$-pole perturbations. The detuning parameter $\theta=0.7$
Figure 4 illustrates the stability of these CLBs against multipole perturbations for $\ell=1,2,3, \ldots, 20$. The eigenvalue $\Lambda_{1}$ is quite evidently null at all intensity values. This feature is a fingerprint of the neutral (or so-called Goldstone) modes $[28,29]$. These modes are a manifestation of the translational invariance of the intracavity dynamics.

To see how this symmetry leads to neutral dipole modes, let us consider a small translation of the stationary field by $\vec{\epsilon}$ with magnitude $|\vec{\epsilon}| \ll 1$, which results in the perturbation $\delta \phi_{\vec{\epsilon}}(\vec{\rho}) \equiv \phi_{S}(\vec{\rho}+\vec{\epsilon})-\phi_{S}(\vec{\rho}) \approx \vec{\epsilon} \cdot \vec{\nabla} \phi_{S}(\vec{\rho})$. For the spherically symmetric CLBs under consideration $\phi_{S}(\vec{\rho})=\phi_{S}\left(\vec{\rho}^{(c)}+\rho \hat{\rho}\right)$ $=\phi_{S}(\rho)$, we have $\vec{\nabla} \phi_{S}(\vec{\rho})=\hat{\rho}\left(d \phi_{S} / d \rho\right)$, and the perturbation becomes $\delta \phi_{\vec{\epsilon}}(\vec{\rho}) \approx \epsilon\left(d \phi_{S} / d \rho\right)(\hat{\epsilon} \cdot \hat{\rho})$, where $\hat{\epsilon} \equiv \vec{\epsilon} /|\vec{\epsilon}|$. From the addition theorem of spherical harmonics [30] we have $(\hat{\boldsymbol{\epsilon}} \cdot \hat{\rho})=(4 \pi / 3) \sum_{m=-1}^{1} Y_{1, m}^{*}(\hat{\epsilon}) Y_{1, m}(\hat{\rho})$. Hence, we can write

$$
\delta \phi_{\vec{\epsilon}}(\vec{\rho}) \approx \epsilon \frac{d \phi_{S}}{d \rho} \frac{4 \pi}{3} \sum_{m=-1}^{1} Y_{1, m}^{*}(\hat{\epsilon}) Y_{1, m}(\hat{\rho}),
$$

which shows that the perturbation associated with a translation is, in fact, a dipole perturbation.

Furthermore, since both $\phi_{S}$ and its translation are stationary, $\partial \delta \phi_{\vec{\epsilon}} / \partial \tau=0$ for $|\vec{\epsilon}| \ll 1$. We have therefore found that $\left(\partial \phi_{S} / \partial \rho, \partial \phi_{S}^{*} / \partial \rho\right)^{T}$ is an eigenvector of $\mathcal{J}_{1}$ with zero eigenvalue. Comparison of this eigenvector with that obtained numerically for the eigenvalue $\Lambda_{1}$ reveals that the latter is indeed a neutral mode arising from the translational symmetry. The CLBs are therefore marginally stable against translations and stable against all other dipole perturbations.

For $\ell=2,3$ and $\ell=7, \ldots, 20$, the stability parameter $\Lambda_{\ell}$ tends to follow that of the homogeneous background $\Lambda_{b g}$ $=I_{0}-1$. This is to be expected for larger $\ell$ because the term $-\ell(\ell+1) / \rho^{2}$ appearing in the Laplacian operator $\mathcal{D}_{\ell}$ acts as a centrifugal potential, pushing multipole perturbations far from the center of the bullet. These disturbances, therefore, would only be influenced by the background field on which the bullet resides. For $\ell=4,5$, and 6 , however, the stability parameters tend to be slightly larger than those of the background; none the less, the CLBs are stable against $2^{4}-, 2^{5}$-, and $2^{6}$-pole disturbances for most background intensities.

As occurs in the propagation of nonlinear Bessel beams through bulk Kerr media (outside of a cavity) [17,18], nonlinear losses can serve to stabilize the CLBs against symmetric perturbations. We have investigated the effects of stabilization for various numbers of absorbed photons $K$.

The stabilizing influence of nonlinear losses are shown in Fig. 5, where we show how a $K=6$-photon absorption process alters the stationary solutions of the upper CLB branch for parameters $\left\{I_{0}=0.9, \theta=0.7\right\}$. Figure 5 (a) shows the peak intensity of the CLB, and Fig. 5(b) demonstrates that nonlinear losses stabilize the bullet against spherically symmetric perturbations. This stabilizing effect is illustrated by the decrease in $\Lambda_{0}$ with increasing $\beta_{K}$. We see that $\Lambda_{0}$ decreases to negative values (in the stable region) and abruptly settles to a nearly constant value, which corresponds to the stability of the homogeneous background $\Lambda_{b g}$. The background, in essence, imposes a floor on $\Lambda_{0}$, since perturbations far from the bullet's peak see only the background field and are unaffected by the CLB. Figure 6 illustrates the effects of this 

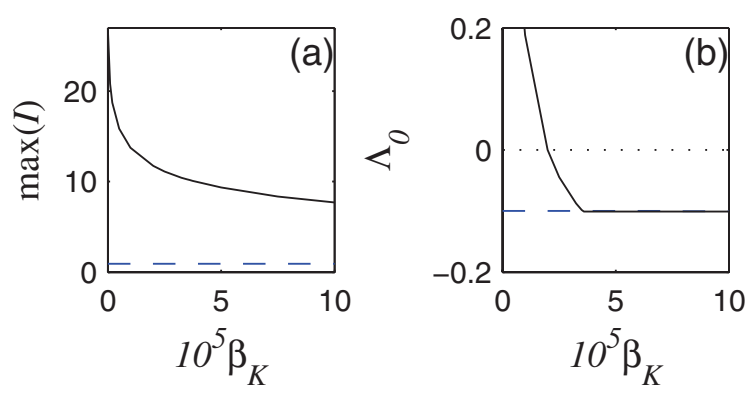

FIG. 5. (Color online) Shows the effects of a $K=6$-photon absorption process on the intensity and symmetric stability properties of a CLB on a homogeneous background with intensity $I_{0}=0.9$, and a detuning parameter $\theta=0.7$. Panel (a) shows the peak intensity of a CLB on the upper branch as a function of the absorption parameter $\beta_{K}$, and panel (b) shows the corresponding stability parameter $\Lambda_{0}$ against spherically symmetric perturbations. The dashed line in panel (a) represents the homogeneous background $I_{0}=0.9$, and the dashed line in panel (b) represents $\Lambda_{b g}$. Both peak intensity and $\Lambda_{0}$ decrease with $\beta_{K}$ and the CLB becomes stable against symmetric perturbations for sufficiently strong absorption.

nonlinear absorption on $2^{\ell}$-pole perturbations for $\ell$ $=1,2, \ldots, 20$. Against these multipole perturbations for $\ell$ $\geq 2$, the nonlinear losses tend to enhance the stability of the CLB. For $\ell=1$, on the other hand, nonlinear losses have no effect on the stability parameter since they do not break the translational invariance of the system.

Preliminary results indicate that some lower-order absorption processes can also stabilize the CLBs, though for higher values of the coupling $\beta_{K}$. For a background intensity $I_{0}$ $=0.9$ and detuning parameter $\theta=0.7$, we have found that $K$-photon absorption processes with $K=4$ and 5 can stabilize CLBs with values of $\beta_{K}$ on the order of $5 \times 10^{-3}$ and 5 $\times 10^{-4}$, respectively. Likewise, higher-order processes can yield stability of CLBs for lower values of $\beta_{K}$. For example,

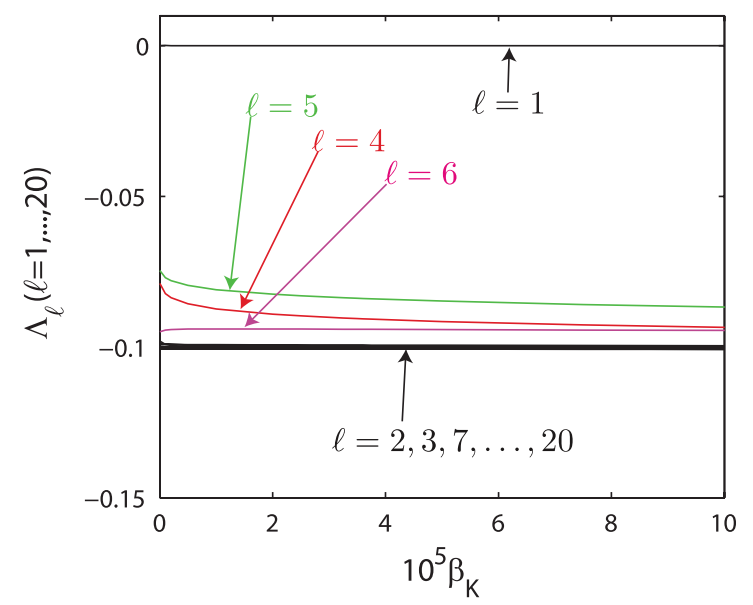

FIG. 6. (Color online) Shows the effects of a $K=6$-photon absorption process on the multipolar stability parameters $\Lambda_{\ell}$ $(\ell=1,2, \ldots, 20)$ of a CLB on a homogeneous background with a normalized intensity $I_{0}=0.9$, and a detuning parameter $\theta=0.7$. Parameters $\Lambda_{4}, \Lambda_{5}$, and $\Lambda_{6}$ decrease with increasing $\beta_{K}$, and $\Lambda_{\ell}$ for $\ell=2,3,7,8, \ldots, 20$ follows $\Lambda_{b g}$. for $K=8$, the CLB is stabilized for $\beta_{K}$ on the order of 5 $\times 10^{-8}$.

\section{DYNAMIC CONTROL OF CLBS: WRITING AND ERASING}

In this section we show through numerical simulations how one can write and erase CLBs at predetermined positions in the cavity. We apply the ideas of the subsection of Sec. II to define control pulse sequences that lead to the formation or destruction of cavity light bullets. As discussed in that section, in order to activate or destroy a CLB centered at position $\vec{\rho}^{(c)}=\vec{\rho}_{\perp}^{(c)}+\zeta^{(c)} \hat{z}$ in the dimensionless moving frame of reference, one needs to apply a sequence of control pulses that addresses this position. These pulses are centered at times $t_{m}^{(c)}=(L / V) m+\left(L-\zeta^{(c)} z_{0}\right) / V \quad(m=0, \pm 1, \ldots) \quad$ [Eq. (10)] and are focused on the input mirror at transverse position $\vec{\rho}_{\perp}^{(c)}$. In this way, every time the target CLB strikes the mirror, a pulse in the sequence will interfere with the CLB and drive its evolution. This pulse train is modulated by a slowly varying function which determines the period over which the sequence is applied. Each sequence, labeled by index (or set of indices) $\nu$, results in driving term $\Upsilon_{\nu}(\vec{\rho}, \tau)$ appearing in the equation of motion for the intracavity field [Eq. (17)]. (A sample input field profile and its corresponding driving term are shown in Fig. 1.)

We first demonstrate through simulations which assume a spherically symmetric field how one can write and subsequently erase a single CLB at a predetermined position $\vec{\rho}^{(c)}$ $=\vec{\rho}_{\perp}^{(c)}+\zeta^{(c)} \hat{z}$ in the moving reference frame. We consider a write and an erase sequence acting on a cavity with background intensity $I_{0}=0.9$, a detuning parameter $\theta=0.7$, and a nonlinear $K=6$-photon absorption parameter $\beta_{K}=10^{-4}$. The write sequence (labeled by index $\nu=w$ ) is intended to activate a CLB at time $\tau^{(w)}=25$ and an erase sequence (labeled by index $\nu=e)$ is meant to destroy this CLB at time $\tau^{(e)}$ $=125$. These sequences manifest themselves in the driving term of Eq. (17) as $\sum_{\nu=w, e} \Upsilon_{\nu}(\vec{\rho}, \tau)$, where we have chosen

$$
\Upsilon_{\nu}(\vec{\rho}, \tau)=A_{\nu} \exp \left[-\frac{\left(\tau-\tau^{(\nu)}\right)^{2}}{4 \sigma_{\tau}^{2}}-\frac{\left|\vec{\rho}-\vec{\rho}^{(c)}\right|^{2}}{4 \sigma_{\rho}^{2}}\right] .
$$

We take the write and erase amplitudes to be $A_{w}=-A_{e}$ $=0.5 \exp \left[i \arg \left(\Upsilon_{0}\right)\right]$ so that the write sequence has the same phase as the holding beam $Y_{0}$ and the erase sequence has the opposite phase. The control sequences both have duration $\sigma_{\tau}=2.5$ and dimensionless widths $\sigma_{\rho}=1.0$. Assuming the intracavity field $\Phi(\vec{\rho}, \tau)=\Phi_{0}[1+\phi(\vec{\rho}, \tau)]$ [where $\Phi_{0}$ is determined by Eq. (18)] is spherically symmetric about the central CLB position $\vec{\rho}^{(c)}$, we solved the dynamic evolution for the field $u(\rho, \tau) \equiv \rho \phi(\rho, \tau)$, which evolves according to

$$
\frac{\partial u}{\partial \tau}=\rho Q\left(\frac{u}{\rho}, \frac{u^{*}}{\rho}\right),
$$

where $Q$ is given in Eq. (22). This simulation was carried out by tracking the spherically symmetric dynamic variables $u\left(\rho_{j}, \tau\right)$ and $u^{*}\left(\rho_{j}, \tau\right)$ evaluated on an $N$-point spherical grid of radius $R,\left\{\rho_{j}=(j-1 / 2) R / N: j=1, \ldots, N\right\}$, using the fourthorder Runge-Kutta interaction picture method derived in Ref. 


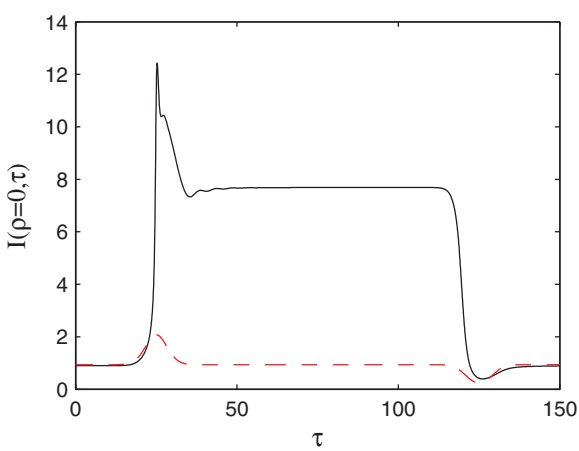

FIG. 7. (Color online) Dynamic simulations demonstrate the process of writing and erasing a CLB. The solid line shows the peak intensity of the CLB as a function of time, while the dashed line indicates the peak intensity of the pump field $|Y(\rho=0, \tau)|^{2}$. The system parameters are the same as those in Figs. 2(c) and 2(d), and the control field's characteristics are described in the text.

[31]. Spatial and temporal resolution parameters, $R=100$ and $N=1024$, as well as the time step, $d \tau=10^{-3}$, were chosen to ensure convergence.

Figure 7 shows the temporal dependence of the peak intensity resulting from this numerical integration. The write sequence, sent in at time $\tau^{(w)}=25$, induces the creation and growth of a localized field superposed on the homogeneous background. The growth is quickly saturated by nonlinear absorption. As the write sequence subsides, the peak amplitude begins to decay. Then, after this initial decay, the peak is refilled due to energy flow from the tails and the cw pump field. Figure 7 shows that the peak intensity then undergoes damped oscillations, and we find that the field profile settles down to the stationary solution depicted in Figs. 2(c) and 2 (d). At time $\tau^{(e)}=125$, the erase sequence with a phase opposite to that of the holding beam destroys the bullet, and the intracavity field reverts back to the homogeneous background.

The addressing procedure discussed in the subsection of Sec. II also allows one to write and erase multiple CLBs at different (longitudinal and transverse) positions independently. We illustrate this capability through full threedimensional dynamic simulations of Eq. (17) in which we consider a cavity of dimensionless length $L / z_{0}=25$. The background intensity, detuning, and nonlinear absorption parameters are the same as those used in the spherically symmetric simulations. The control fields involved in this simulation address locations $\vec{\rho}^{(1)}=6.25 \hat{z}$ and $\vec{\rho}^{(2)}=14.25 \hat{z}$. Two write sequences activate CLBs at positions $\vec{\rho}^{(1)}$ and $\vec{\rho}^{(2)}$ at times $\tau^{(1, w)}=25$ and $\tau^{(2, w)}=75$, respectively. At later times, we send in erase sequences with opposite phase at those same locations: we turn off the CLB formed at $\vec{\rho}^{(1)}$ at time $\tau^{(1, e)}=125$, and that formed at $\vec{\rho}^{(2)}$ was erased at time $\tau^{(2, e)}$ $=175$. These four control sequences manifest themselves in the driving term of Eq. (17) as $\sum_{i=1}^{2} \Sigma_{v \in\{w, e\}} \Upsilon_{i, v}(\vec{\rho}, \tau)$, where each sequence contributes a term of the form

$$
Y_{i, v}(\vec{\rho}, \tau)=A_{v} \exp \left[-\frac{\left(\tau-\tau^{(i, v)}\right)^{2}}{4 \sigma_{\tau}^{2}}-\frac{\left|\vec{\rho}-\vec{\rho}^{(i)}\right|^{2}}{4 \sigma_{\rho}^{2}}\right],
$$

and the spatial and temporal widths are $\sigma_{\rho}=1.0$ and $\sigma_{\tau}=2.5$,
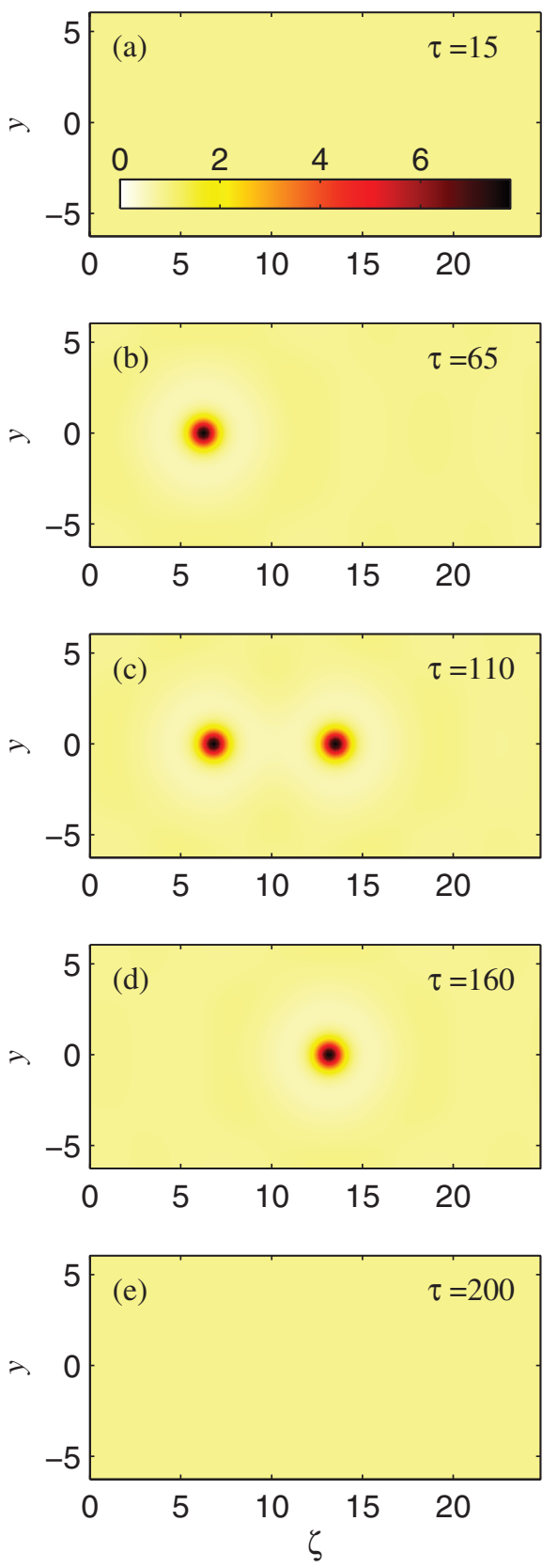

FIG. 8. (Color online) cross sections of the intracavity intensity profile $I(x=0, y, \zeta, \tau)$ at various times resulting from the write and erase sequences described by Eq. (29). The intensity scale is shown in the inset of panel (a). Two CLBs are written to the cavity at positions $\vec{\rho}^{(1)}=6.25 \hat{z}$ and $\vec{\rho}^{(2)}=14.25 \hat{z}$ at times $\tau^{(1, w)}=25$ and $\tau^{(2, w)}$ $=75$, respectively. They are then subsequently erased at respective times $\tau^{(1, e)}=125$ and $\tau^{(2, e)}=175$. The simulation and cavity parameters are given in the text.

respectively. As with the symmetric simulations, we chose the amplitudes that multiplies the temporal modulating Gaussian to be $A_{w}=-A_{e}=0.5 \exp \left[i \arg \left(\Upsilon_{0}\right)\right]$, and the duration of this Gaussian $\sigma_{\tau}=2.5$.

A cross section of the intensity profile $I(x=0, y, \zeta, \tau)$ that results from these write and erase sequences is shown for various times in Fig. 8. Here, as usual, $x$ and $y$ are the trans- 
verse dimensionless coordinates. The numerical integration was carried out on an $N_{x} \times N_{y} \times N_{\zeta}$ Cartesian grid with longitudinal length $L=25 z_{0}$ and transverse lengths $L_{x}=L_{y}$ $=12.5 r_{0}$, where $N_{x}=N_{y}=64$ are the number of grid points along the transverse directions, and $N_{\zeta}=128$ is the number of points along the longitudinal direction. Although boundary effects cannot be completely ignored, these simulations illustrate how the control fields can address and control CLBs at distinct positions. Panel (a) shows the homogeneous field intensity at $\tau=15$ before the first CLB is written, while panel (b) illustrates the intracavity intensity at a time, $\tau=65$, well after the first bullet has formed. The field at $\tau=110$ is shown in panel (c), where we see that two CLBs were successfully written to the cavity. While both CLBs are active in the cavity, they do move closer together by a fraction of a CLB length. The movement, however, is negligible and does not influence the efficacy of the erase sequences. This motion results from the influence the tail of each bullet has on the peak of the other. Because the bullet's tails fall off exponentially with the distance from its center, this effective interaction would vanish for CLBs written farther apart from one and other. The intensity profile after the first CLB is erased is shown in panel (d), and the profile after both bullets are erased is shown in panel (e).

\section{CONCLUSION}

In conclusion, we have shown how dynamics within a low loss ring cavity can permit three-dimensional localized solutions in the presence of a Kerr medium.

In Sec. III, we found localized stationary solutions to the intracavity field evolution equations. Though these solutions are unstable, we have shown that multiphoton absorption serves to stabilize the solutions. We also examined the intensity and spectral profiles of these bullets, and found a qualitative similarity to freely propagating $O$ waves, which may also form in the presence of nonlinear losses [18].

In Sec. IV, we have shown through numerical simulations how one can write and erase CLBs at specific points in the moving frame of reference. These cavity light bullets could serve as elements in controllable three-dimensional optical pixel arrays.

\section{ACKNOWLEDGMENTS}

We would like to thank D. Faccio, M. Clerici, and P. Di Trapani for useful discussions. We are grateful to an anonymous referee for suggesting that the neutral modes we found through numerical linear stability analysis are actually Goldstone modes associated with translational invariance. This work was supported by the European STREP Project No. 004868 (FunFACS).
[1] Y. Silberberg, Opt. Lett. 15, 1282 (1990).

[2] L. A. Lugiato and R. Lefever, Phys. Rev. Lett. 58, 2209 (1987).

[3] W. J. Firth and A. Lord, J. Mod. Opt. 43, 1071 (1996).

[4] W. J. Firth and A. J. Scroggie, Phys. Rev. Lett. 76, 1623 (1996).

[5] L. Spinelli, G. Tissoni, M. Brambilla, F. Prati, and L. A. Lugiato, Phys. Rev. A 58, 2542 (1998).

[6] W. J. Firth, G. K. Harkness, A. Lord, J. M. McSloy, D. Gomila, and P. Colet, J. Opt. Soc. Am. B 19, 747 (2002).

[7] X. Hachair et al., IEEE J. Sel. Top. Quantum Electron. 12, 339 (2006).

[8] M. Brambilla, L. A. Lugiato, and M. Stefani, Europhys. Lett. 34, 109 (1996).

[9] G. S. McDonald and W. Firth, J. Opt. Soc. Am. B 10, 1081 (1993).

[10] M. Saffman, D. Montgomery, and D. C. Anderson, Opt. Lett. 19, 518 (1994).

[11] V. B. Taranenko, K. Staliunas, and C. O. Weiss, Phys. Rev. A 56, 1582 (1997).

[12] C. O. Weiss, M. Vaupel, K. Staliunas, G. Slekys, and V. B. Taranenko, Appl. Phys. B: Lasers Opt. 68, 151 (1999).

[13] S. Barland et al., Nature (London) 419, 699 (2002).

[14] X. Hachair et al., Phys. Rev. A 69, 043817 (2004).

[15] M. Brambilla, T. Maggipinto, G. Patera, and L. Columbo, Phys. Rev. Lett. 93, 203901 (2004).

[16] M. Tlidi, M. Haelterman, and P. Mandel, Quantum Semiclass. Opt. 10, 869 (1998).
[17] M. A. Porras, A. Parola, D. Faccio, A. Dubietis, and P. Di Trapani, Phys. Rev. Lett. 93, 153902 (2004).

[18] M. A. Porras, A. Parola, and P. Di Trapani, J. Opt. Soc. Am. B 22, 1406 (2005).

[19] A. Dubietis, E. Gaižauskas, G. Tamošauskas, and P. Di Trapani, Phys. Rev. Lett. 92, 253903 (2004).

[20] M. A. Porras, A. Dubietis, E. Kučinskas, F. Bragheri, V. Degiorgio, A. Couairon, D. Faccio, and P. Di Trapani, Opt. Lett. 30, 3398 (2005).

[21] A. Dubietis, A. Couairon, E. Kučinskas, G. Tamošauskas, E. Gaižauskas, D. Faccio, and P. Di Trapani, Appl. Phys. B: Lasers Opt. 84, 439 (2006).

[22] D. Faccio et al., Opt. Express 16, 8213 (2008).

[23] L. A. Lugiato, in Progress in Optics, edited by E. Wolf (Elsevier/North-Holland, Amsterdam, 1984), Vol. 21, pp. 69 216.

[24] A. C. Newell and J. V. Moloney, Nonlinear Optics (AddisonWesley, California, 1992), Chap. 2.

[25] L. A. Lugiato and C. Oldano, Phys. Rev. A 37, 3896 (1988).

[26] D. Lemoine, J. Chem. Phys. 101, 3936 (1994).

[27] D. Lemoine, Comput. Phys. Commun. 99, 297 (1997).

[28] W. J. Firth and G. K. Harkness, Asian J. Phys. 7, 665 (1998).

[29] T. Maggipinto, M. Brambilla, G. K. Harkness, and W. J. Firth, Phys. Rev. E 62, 8726 (2000).

[30] M. E. Rose, Elementary Theory of Angular Momentum (Dover, New York, 1957).

[31] B. M. Caradoc-Davies, Ph.D. thesis, University of Otago, 2000 (unpublished). 